\title{
Prevention of incisional hernia using different suture materials for closing the abdominal wall: a comparison of PDS, Vicryl and Prolene in a rat model
}

\author{
S. van Steensel ${ }^{1,2}$ (D) - L. C. L. van den $\mathrm{Hil}^{1,2} \cdot$ A. Bloemen $^{3} \cdot$ M. J. Gijbels ${ }^{4} \cdot$ S. O. Breukink ${ }^{1,2} \cdot$ J. Melenhorst $^{1}$. \\ K. Lenaerts ${ }^{1,2} \cdot$ N. D. Bouvy ${ }^{1,2}$
}

Received: 30 January 2019 / Accepted: 26 March 2019 / Published online: 20 May 2019

(c) The Author(s) 2019

\begin{abstract}
Purpose An incisional hernia occurs frequently after a midline incision with an incidence of $12.8 \%$. The choice in suture material used for abdominal wall closure is not straightforward and the conflicting literature focuses on clinical outcomes. This study compares a non-absorbable, slow-absorbable and fast-absorbable suture in a rat model, focusing on histological outcomes predicting better fascia healing.

Methods 33 male Wistar rats, divided over three groups, each received two separate $1 \mathrm{~cm}$ incisions closed with either Prolene 4/0, PDS 4/0 or Vicryl 4/0. At 7 days and 21 days, one of the incisions was explanted. Tissue was semi-quantitatively scored regarding inflammatory cells and collagen fibres present. Using qPCR macrophage polarisation, fibroblast activity and vascularisation were evaluated. Data were analysed by Kruskal-Wallis test with Mann-Whitney $U$ post hoc test. A $p$ value of 0.017 was considered significant after Bonferroni correction.

Results All animals recovered without complications and completed the 21 days of follow-up. The Vicryl group showed a higher presence of macrophages after 21 days in comparison with Prolene $(p=0.003)$ and PDS $(p=0.006)$ and more foreign body giant cells compared to Prolene at 7 days $(p=0.010)$ and PDS at 21 days $(p<0.001)$. qPCR showed 2.5 -fold higher expression of clec10A in PDS compared to Prolene after 7 days $(p=0.007)$.

Conclusions The results of this study carefully support the use of PDS suture, compared to Prolene and Vicryl, in abdominal wall closure based on a favourable macrophage response. The heterogeneity and variability in the data might be explained by the spectrum of the macrophage subtype paradigm.
\end{abstract}

Keywords Incisional hernia $\cdot$ Prevention $\cdot$ Suture $\cdot$ PDS $\cdot$ Vicryl $\cdot$ Prolene $\cdot$ Rat model $\cdot$ Abdominal wall

Electronic supplementary material The online version of this article (https://doi.org/10.1007/s10029-019-01941-9) contains supplementary material, which is available to authorized users.

N. D. Bouvy

n.bouvy@mumc.nl

1 Department of General Surgery, Maastricht University Medical Centre, P. O. Box 5800, 6202 AZ Maastricht, The Netherlands

2 NUTRIM School of Nutrition and Translational Research in Metabolism, Maastricht University, Maastricht, The Netherlands

3 Department of General Surgery, VieCurie Medical Centre, Venlo, The Netherlands

4 Department of Pathology, Maastricht University Medical Centre, Maastricht, The Netherlands

\section{Introduction}

An incisional hernia is a frequent complication following abdominal surgery and is defined as any abdominal wall gap with or without a bulge in the area of a postoperative scar perceptible or palpable by clinical examination or imaging $[1,2]$. Bulging, discomfort or pain and cosmetic issues have a significant impact on the quality of life of patients [3].

At a weighted mean follow-up of 23.7 months, 12.8\% of the patients have developed an incisional hernia and the estimated risk to undergo an incisional hernia repair after a midline incision is $5.2 \%$ [4]. In high-risk patients, the incidence increases to up to $30 \%[5,6]$. The identified patient-related risk factors are obesity (BMI $>25 \mathrm{~kg} / \mathrm{m}^{2}$ ), the presence of an abdominal aortic aneurysm and congenital connective tissue disorders [7-12]. Furthermore, 
postoperative wound complications are an independent risk factor for the development of an incisional hernia [9].

With unacceptable high recurrence rates after incisional hernia repair, which ranges from $23.8 \%$ to $32 \%$ [13-16], a preventive strategy has become the focus of scientific research. In high-risk patients, preventive mesh placement proves an effective tool in reducing incisional hernia rates $[6,17,18]$. However, if the mesh is placed intra-peritoneally, delayed wound healing and increased pain after 6 week follow-up are observed [19]. Thus, improving the technique of primary abdominal wall closure might be advantageous.

The technique for closure of the abdominal wall after a midline incision has evolved from using interrupted sutures to a continuous running monofilament suture technique $[20,21]$. Also, a small stitch suture technique, with a $4: 1$ suture length to wound length ratio, decreases the incidence of incisional hernia [22, 23]. The European Hernia Society formulated a guideline for the closure of the abdominal wall incorporating the previous statements. This guideline makes a weak recommendation for abdominal wall closure with slow-absorbable sutures, which is adopted in standard clinical practice [24]. Multiple metaanalyses report conflicting results regarding the preferable suture material, caused by substantial heterogeneity between studies and inclusion criteria [4, 20, 21, 25].

Two meta-analyses suggested the use of slow-absorbable sutures based on a lower occurrence of suture sinuses and wound pain, but in the absence of a difference in incisional hernia occurrence $[21,25]$. The most recent metaanalysis recommended slow-absorbable sutures based on a decrease of incisional hernia compared to fast-absorbable sutures, but a separate comparison between only slowabsorbable and non-absorbable sutures was not performed [20]. Lastly, Bosanquet et al. suggested in a meta-regression that suture material does not have influence on incisional hernia rate or on the occurrence of suture sinuses [4].

Collagen metabolism plays a central role in the healing of the abdominal wall. Collagen maturation and collagen breakdown in particular are crucial. A decreased collagen I/III ratio is indicative of a low presence of mature collagen. Also increased MMP activity, responsible for collagen type I denaturation, is involved in abdominal hernia development [26]. However, the healing process of the abdominal wall is still not fully understood. This unknown factor complicates the discussion regarding incisional hernia prevention. The aim of this study is to investigate the effect of different types of suture materials on the healing of the abdominal wall in a rat model. Giving insight to physiological and pathophysiological processes might lead to new starting points for preventive strategies.

\section{Materials and methods}

The protocol was approved by the animal ethics committee of the Maastricht University conform the Dutch Experiments on Animals Act.

\section{Materials}

A non-absorbable monofilament polypropylene suture (Prolene 4/0, Ethicon Inc; Johnson \& Johnson, Somerville, NJ, USA), a slow-absorbable monofilament polydioxanone suture (PDS II 4/0, Ethicon Inc; Johnson \& Johnson, Somerville, NJ, USA), and a fast-absorbable multifilament polyglactine suture (Vicryl 4/0, Ethicon Inc; Johnson \& Johnson, Somerville, NJ, USA) were obtained commercially.

\section{Study design}

33 male Wistar rats between 240 and $260 \mathrm{~g}$ were acquired from a registered breeding facility (Envigo, Horst, the Netherlands), housed at the Maastricht University animal facilities and cared for according to local protocol. The animals were socially housed in filter-topped cages with a $12 \mathrm{~h}$ daynight cycle and had free access to food and water. Because of hormonal influences on wound healing by progesterone and oestrogen [27] and a faster postoperative weight recovery of males compared to females [28], only male rats were used in this experiment. The animals were randomly assigned to one of the three groups for abdominal wall closure of two separate midline incisions by either Prolene $4 / 0$, PDS $4 / 0$ or Vicryl 4/0. There were two time points for tissue evaluation, after 7 days and 21 days of follow-up.

\section{Procedure}

After a 1-week acclimatisation period, preoperative pain medication (buprenorphine $0.05 \mathrm{mg} / \mathrm{kg}$ ) was administered. Anaesthesia was induced using 3-4\% isoflurane and maintained with $2.5 \%$ isoflurane through inhalation of an air mixture. The animal was placed on a sterile field after the abdomen was shaved and the skin was disinfected with $2 \%$ iodine solution.

Via a midline incision of $6 \mathrm{~cm}$ in the skin, the abdominal wall was exposed. Two smaller full-thickness incisions of approximately $1 \mathrm{~cm}$ were made in the midline of the exposed abdominal wall, with a minimal distance of $2 \mathrm{~cm}$ between the two incisions. The two incisions were closed with either Prolene 4/0, PDS 4/0 or Vicryl 4/0 using a continuous suture technique; the skin was closed with Monocryl 4/0 (Ethicon 
Table 1 All primers were tested for transcription of the intended gene

\begin{tabular}{|c|c|c|c|c|}
\hline Gene name & Product length & & GC $\%$ & Sequence \\
\hline \multirow[t]{2}{*}{ rplp0 (ribosomal protein lateral stalk subunit $\mathrm{P} 0$ ) } & 190 & f & 55.00 & CCTCACCGAGATTAGGGACA \\
\hline & & $\mathrm{r}$ & 45.00 & ATCGCTCAGGATTTCAATGG \\
\hline \multirow[t]{2}{*}{$a c t b$ (actin, beta) } & 297 & f & 55.00 & CCGCGAGTACAACCTTCTTG \\
\hline & & $\mathrm{r}$ & 55.00 & CAGTTGGTGACAATGCCGTG \\
\hline \multirow[t]{2}{*}{ il6 (Interleukin 6) } & 246 & $\mathrm{f}$ & 57.14 & CTCTCCGCAAGAGACTTCCAG \\
\hline & & $\mathrm{r}$ & 47.62 & TTCTGACAGTGCATCATCGCT \\
\hline \multirow[t]{2}{*}{ nos2 (iNOS) } & 234 & $\mathrm{f}$ & 52.38 & TAGTCAACTACAAGCCCCACG \\
\hline & & $\mathrm{r}$ & 60 & GTGAGGAACTGGGGGAAACC \\
\hline \multirow[t]{2}{*}{ cd86 (CD86) } & 164 & $\mathrm{f}$ & 45.45 & AGACATGTGTAACCTGCACCAT \\
\hline & & $\mathrm{r}$ & 55 & TACGAGCTCACTCGGGCTTA \\
\hline \multirow[t]{2}{*}{ illo (Interleukin 10) } & 186 & $f$ & 52.38 & CGACGCTGTCATCGATTTCTC \\
\hline & & $\mathrm{r}$ & 60.00 & CAGTAGATGCCGGGTGGTTC \\
\hline \multirow[t]{2}{*}{ clec10a (C-type lectin domain containing 10a) } & 164 & $\mathrm{f}$ & 60.00 & GAGGCTTGAGCCAGAAGGTG \\
\hline & & $\mathrm{r}$ & 52.38 & TGCTGAGCCGTTGTTCTTGAG \\
\hline \multirow[t]{2}{*}{ mrcl (mannose receptor $\mathrm{C}$ typ 1$)$} & 212 & $\mathrm{f}$ & 60.00 & CCCGCTCCTCAAGACAATCC \\
\hline & & $\mathrm{r}$ & 55.00 & AAATACGGTGACTGCCCACC \\
\hline \multirow[t]{2}{*}{$c d 163(\mathrm{CD} 163)$} & 131 & $\mathrm{f}$ & 60 & CTCTGAAGCGACGACAGACC \\
\hline & & $\mathrm{r}$ & 50 & ATGCCAACCCGAGGATTTCA \\
\hline \multirow[t]{2}{*}{$\operatorname{tg} f b 1$ (transforming growth factor- $\beta$ ) } & 115 & f & 60.00 & GGCTGAACCAAGGAGACGGA \\
\hline & & $\mathrm{r}$ & 55.00 & CCTCGACGTTTGGGACTGAT \\
\hline \multirow[t]{2}{*}{ vegfa (vascular endothelial growth factor a) } & 235 & f & 60 & AGAAGGGGAGCAGAAAGCCC \\
\hline & & $\mathrm{r}$ & 47.83 & GATCCGCATGATCTGCATAGTGA \\
\hline \multirow[t]{2}{*}{ angpt2 (angiopoietin 2) } & 168 & $\mathrm{f}$ & 55 & CATGATGTCATCGCCCGACT \\
\hline & & $\mathrm{r}$ & 52.38 & TCCATGTCACAGTAGGCCTTG \\
\hline \multirow[t]{2}{*}{ nos3 (eNOS) } & 139 & f & 52.38 & GAATGGAGAGAGCTTTGCAGC \\
\hline & & $\mathrm{r}$ & 60 & CCGCCAAGAGGATACCAGTG \\
\hline \multirow[t]{2}{*}{ collal (collagen type 1 alpha 1 chain) } & 237 & $\mathrm{f}$ & 60 & CTGACTGGAAGAGCGGAGAG \\
\hline & & $\mathrm{r}$ & 55.00 & CAGGATCGGAACCTTCGCTT \\
\hline \multirow[t]{2}{*}{ mmpl (matrix metallopeptidase 1) } & 144 & f & 55.00 & AAGGCCACTGGTGATCTTGC \\
\hline & & $\mathrm{r}$ & 43.48 & GGTATTTCCAGACTGTTTCCACA \\
\hline \multirow[t]{2}{*}{ fnl (Fibronectin 1) } & 165 & $\mathrm{f}$ & 63.16 & TCСССТCCCAGAGAAGTGG \\
\hline & & $\mathrm{r}$ & 43.48 & TTGGGGAAGCTCATCTGTCTTTT \\
\hline
\end{tabular}

Inc; Johnson \& Johnson, Somerville, NJ, USA). Postoperatively, the animals were administrated fluid resuscitation and recovered under a heat lamp.

After 7 days of follow-up, one of the closed midline incisions was explanted and after 21 days of follow-up the second previously closed midline incision was explanted after killing, using the operative procedure as described above. Using the previous midline incision, randomly either the upper or lower abdominal wall incision was explanted equally distributed over the experimental groups. The explanted specimens were cut in half and one-half was preserved in liquid nitrogen and the other half in formaldehyde $4 \%$ for further analysis. The animals were killed by carbon dioxide overdose at the completion of the follow-up at 21 days.

\section{Histology}

Half of the explanted incision was fixed in $4 \%$ formaldehyde, dehydrated and embedded in paraffin. Subsequently, $4 \mu \mathrm{m}$ thick tissue sections were cut and a haematoxylin-eosin staining was performed. An experienced animal pathologist, who was blinded to the group allocation, evaluated the stained sections microscopically. To compare inflammation and collagen deposition, granulocytes, macrophages, foreign body giant cells and collagen fibres were scored using a fourpoint semi-quantitative scoring system (not present, slightly present, moderately present or abundantly present) [29-32]. 


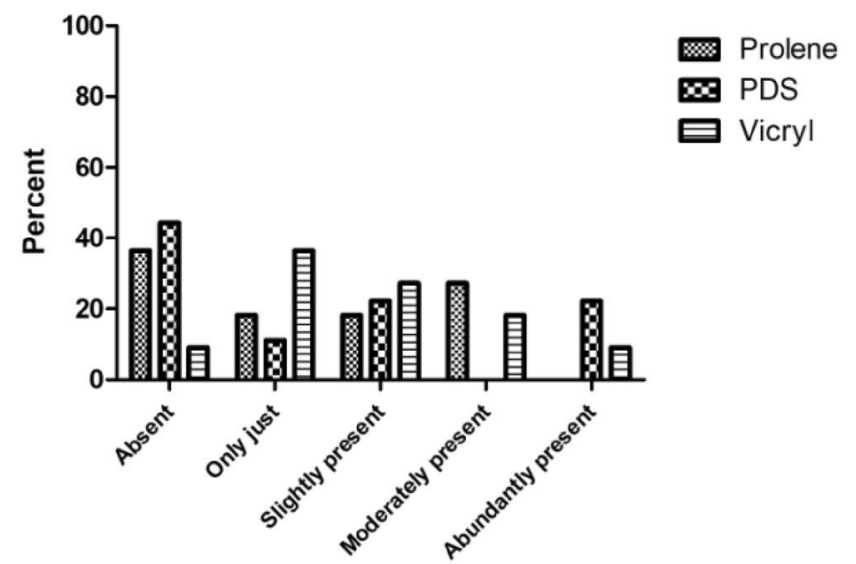

Granylocyte presence at 7 days follow-up

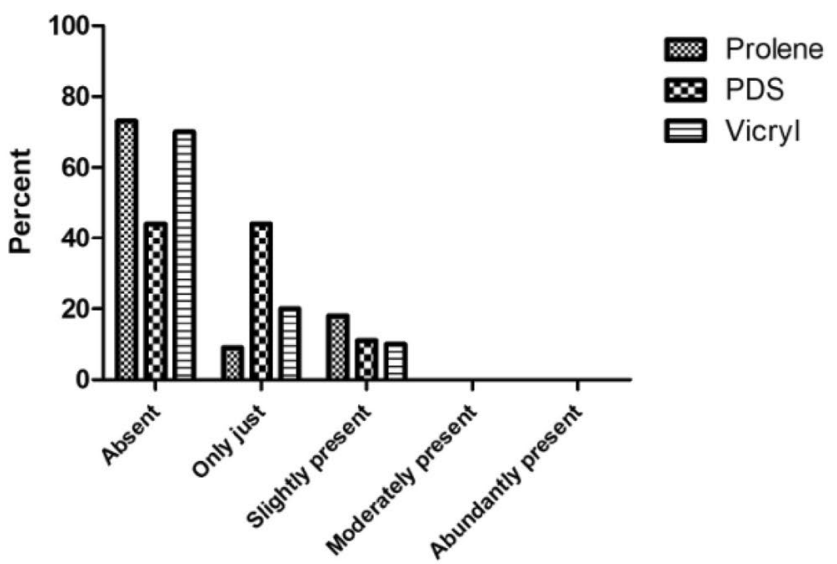

Granylocyte presence at 21 days follow-up

Fig. 1 Semi-quantitative scoring of the presence of granulocytes at 7 days and 21 days follow-up per suture type. At 7 days: $p=0.621$, at 21 days: $p=0.539$

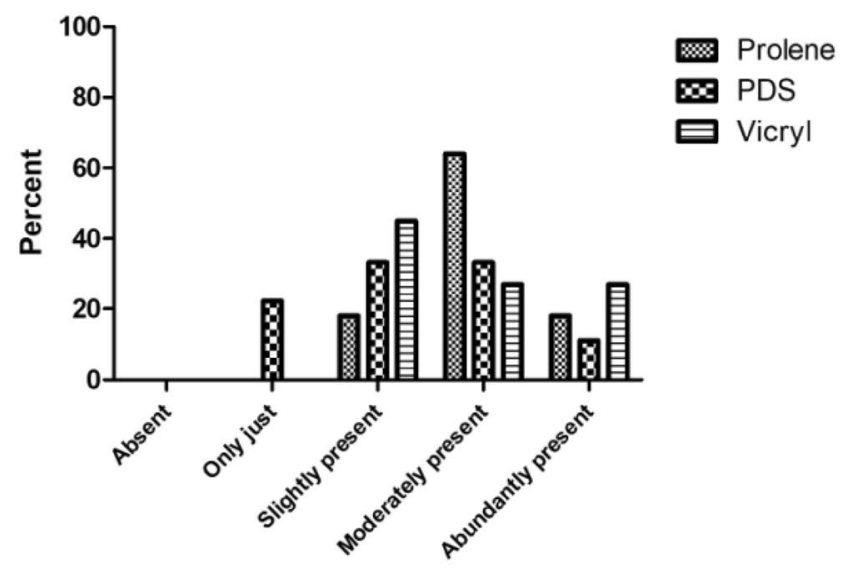

Macrophage presence at 7 days follow-up

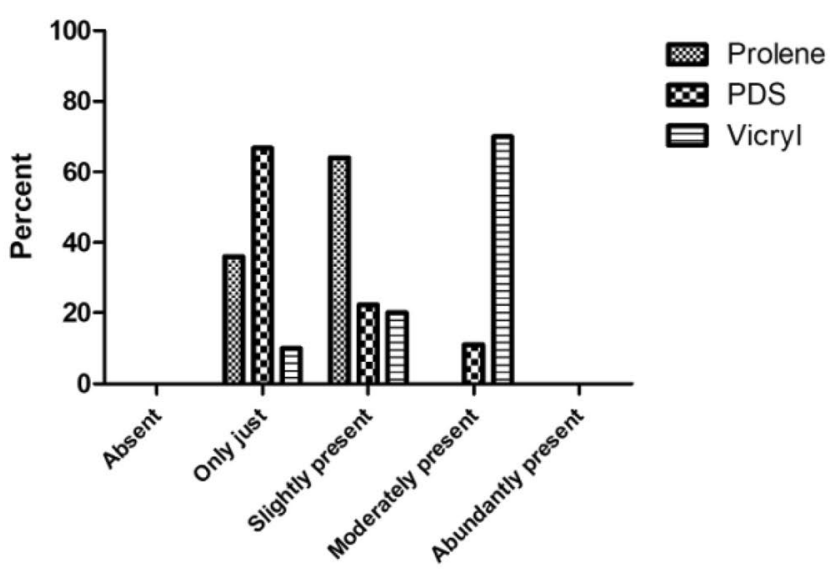

Macrophage presence at 21 days follow-up

Fig. 2 Semi-quantitative scoring of the presence of macrophages at 7 days and 21 days follow-up per suture type. At 7 days: $p=0.257$, at 21 days: Prolene vs Vicryl $p=0.006$, PDS vs Vicryl $p=0.006$, Prolene vs PDS NS

\section{RNA isolation and quantitative real time PCR}

Total RNA was isolated from the snap-frozen abdominal wall specimen using TRI reagent (Sigma, NL). $750 \mathrm{ng}$ DNAse-treated RNA was used to synthesise cDNA (SensiFAST ${ }^{\mathrm{TM}}$, cDNA synthesis kit, Bioline, London, UK). For qPCRs, a volume of $10 \mu \mathrm{l}$ consisting of the cDNA equivalent of $2.5 \mathrm{ng}$ total RNA, $1 \times$ Absolute qPCR SYBR Green Fluorescein Mix (SensiFAST ${ }^{\mathrm{TM}} \mathrm{SYBR}^{\circledR}$ Hi-ROX Kit, Bioline, London, UK) and $0.15 \mu \mathrm{M}$ of gene-specific primers (Sigma, NL) was used (Supplementary Table 1). The LightCycler ${ }^{\circledR}$ 480 Instrument II (Roche Molecular Systems, Inc., was used to perform the qPCR. LinRegPCR software was used to establish gene expression levels. The geometric mean of two internal control genes, Rplp0 and beta-actin (Actb), was calculated and used as normalisation factor. In one sample in the Vicryl group, insufficient cDNA was available for analysis with Actb, resulting in unreliable data. Rplp0 showed no expression in one sample from the Prolene group. For both samples, one reliable housekeeping gene was available, which was used as normalisation factor. Relevant primers were identified from the literature and build using a primer designing tool (Primer-blast) [33]; the sequences are reported in Table 1.

\section{Statistical analysis}

A sample-size calculation was performed in preparation of the experiment. A difference of $20 \%$ in inflammation on a histological level was considered relevant, with a variance of 


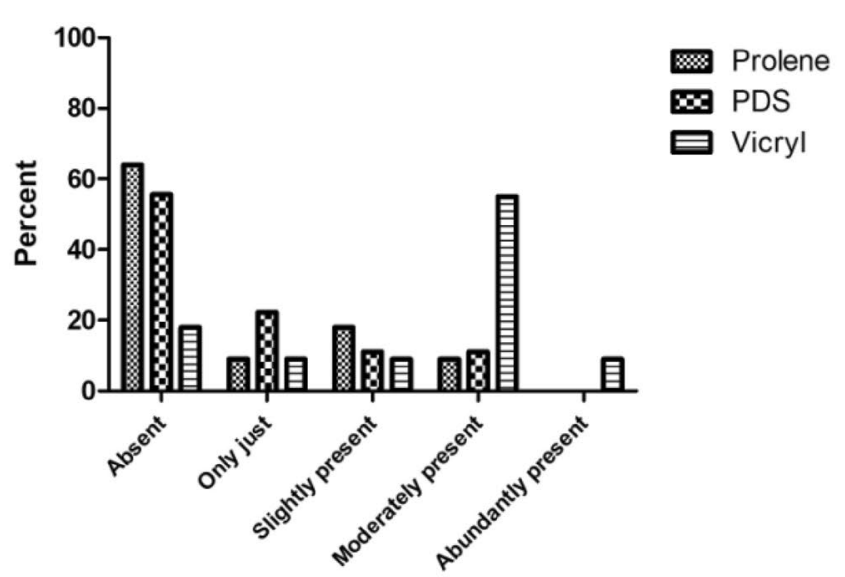

Foreign-body giant cell presence at 7 days follow-up

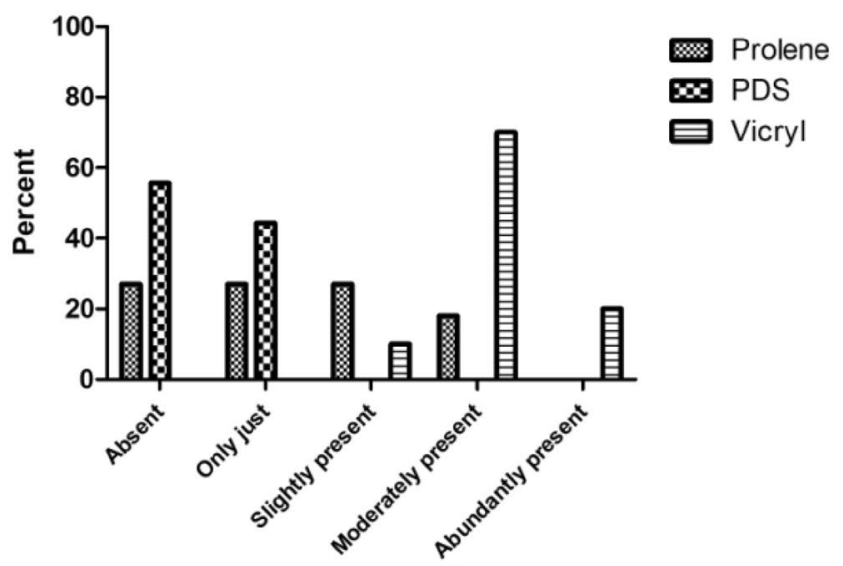

Foreign-body giant cell presence at 21 days follow-up

Fig. 3 Semi-quantitative scoring of the presence of foreign body giant cells at 7 days and 21 days follow-up per suture type. At 7 days: Vicryl vs Prolene $p=0.010$, Vicryl vs PDS NS, Prolene vs PDS NS; at 21 days: Vicryl vs Prolene $p=0.001$, Vicryl vs PDS $p<0.001$

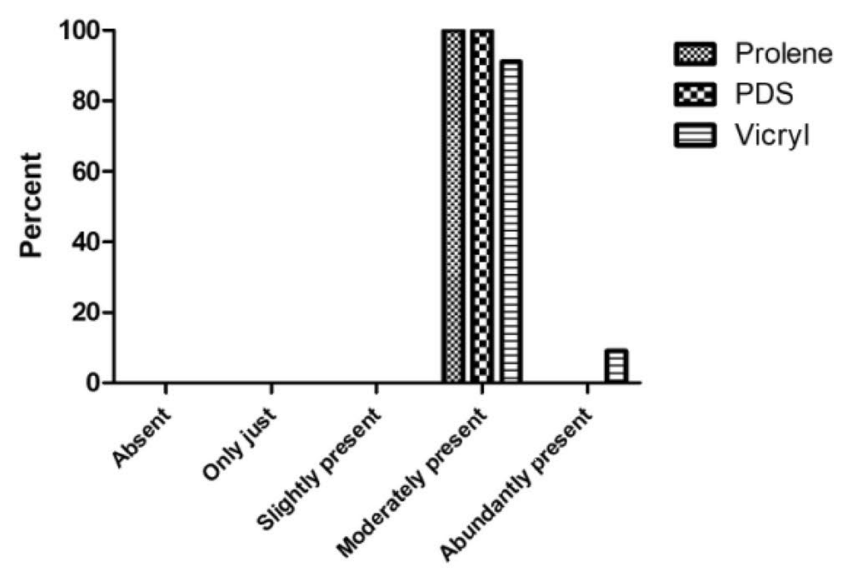

Collagen presence at 7 days follow-up

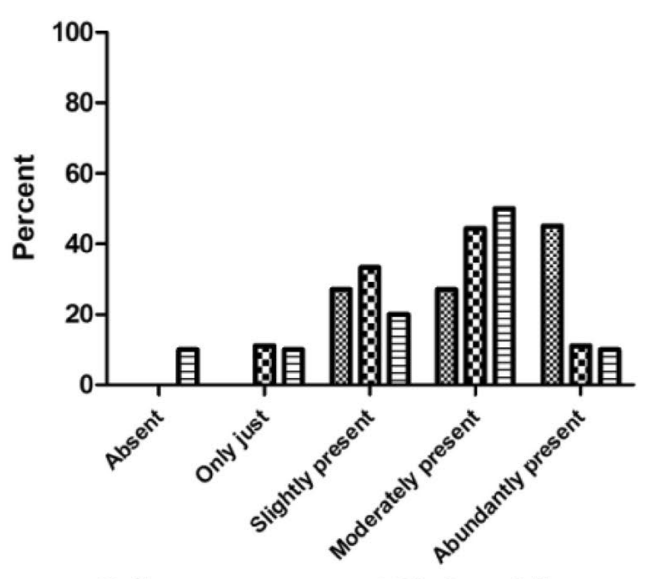

Collagen presence at 21 days follow-up

Fig. 4 Semi-quantitative scoring of the presence of collagen at 7 days and 21 days follow-up per suture type. At 7 days: $p=0.403$, at 21 days: $p=0.202$

$\pm 16 \%$. Alpha was chosen at 0.05 and with a power of 0.80 , resulting in a needed group size of 11 animals per group.

All data were expressed as a median with range or mean with $95 \%$ confidence interval. Nonparametric tests were performed using a Kruskal-Wallis test. In case of significance, a Mann-Whitney $U$ post hoc test was performed to identify specific differences between the groups. A Bonferroni correction was used to correct for multiple testing, so a $p$ value of $0.017(0.05 / 3)$ was considered significant. SPSS 23.0 for Mac (SPSS Inc., Chicago, IL, USA) was used for the statistical analysis.

\section{Results}

Recovery was uncomplicated for all rats. No postoperative complications were encountered, and all animals completed the 21 days of follow-up.

\section{Histology}

For the histological evaluation, 31 out of 33 samples at 7 days and 30 out of 33 samples at 21 days were available for analysis. Two samples in the PDS group were missing for both time points and one sample for the Vicryl group at 21 days of follow-up. 


\section{cd86}
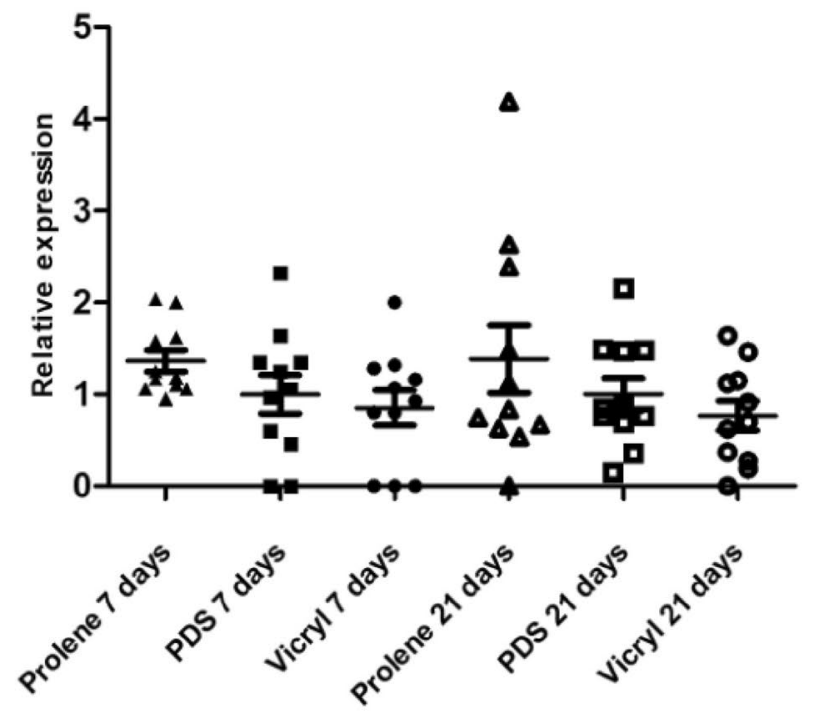

II6

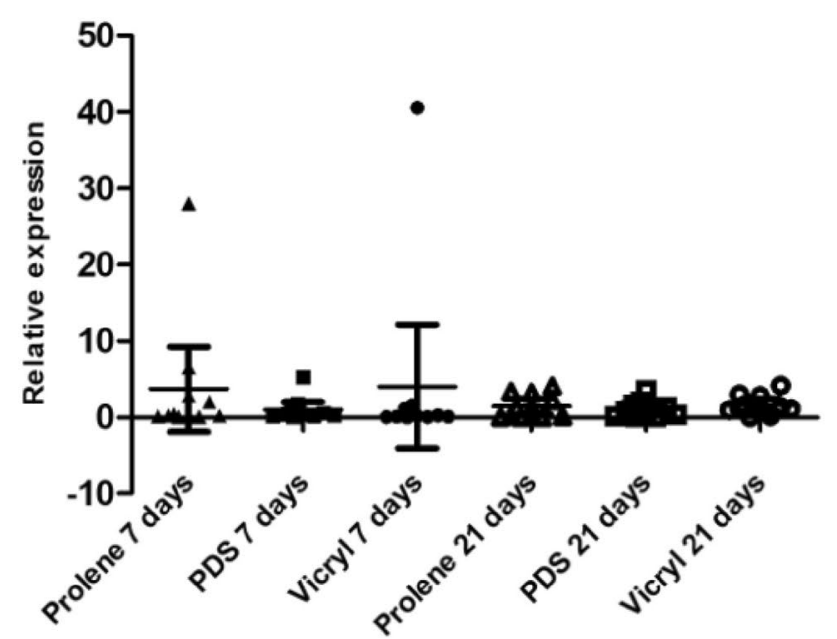

nos2

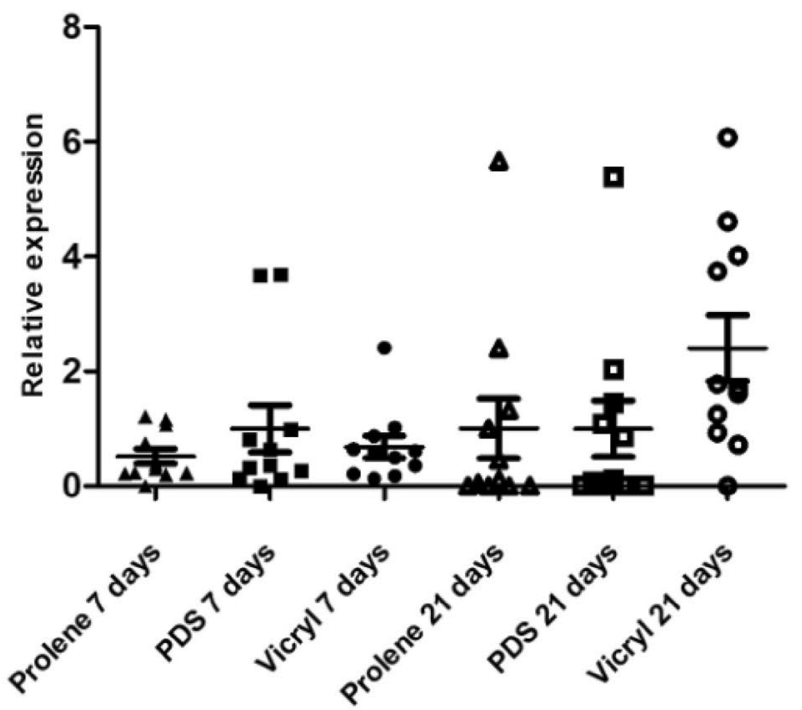

Fig. 5 Scatter plots of relative expression of genes typically expressed by macrophage subtype 1 (cd86, nos 2 and il6) at 7 days and 21 days follow-up. The expression in the Vicryl and Prolene groups is depicted in relation to the PDS group, which was set as the norm

The presence of granulocytes ranged from absent to abundantly present at 7 days of follow-up (Fig. 1a). The presence of granulocytes declined at 21 days of follow-up to a score of slightly present (Fig. 1b). At 7 days and 21 days, the differences between the three groups were not significant, $p=0.621$ and $p=0.539$, respectively. Macrophages were slightly to abundantly present at seven days of follow-up, without significant differences between the groups $(p=0.257)$. The overall presence of macrophages decreased but remained significantly higher in the Vicryl group compared to both Prolene $(p=0.003)$ and PDS $(p=0.006)$ at 21 days of follow-up. The presence of macrophages did not differ significantly between Prolene and PDS after 21 days $(p=0.324)$ (Fig. 2). The Vicryl group showed the highest presence of foreign body giant cells at both time points compared to both Prolene $(p=0.010)$ at both 7 days and 21 days $(p=0.001)$ and compared to PDS at 21 days $(p<0.001)$. At 7 days follow-up, no significant difference was detected 
between Vicryl and PDS regarding foreign body giant cells $(p=0.021)$. Furthermore, Prolene and PDS were comparable regarding the presence of foreign body giant cells at both time points ( $p=0.830$ and 0.053 , respectively) (Fig. 3).

Collagen was moderately present at 7 days in almost all samples $(p=0.403)$. After 21 days, no significant difference was detected between the three groups regarding the presence of collagen ( $p=0.202)$ (Fig. 4).

\section{qPCR}

No samples were excluded in the gene expression analysis. Expression of genes in the PDS group was set as the norm and the expression in the Prolene and Vicryl groups was presented in relation to set norm.

\section{Macrophage polarisation}

Two macrophage subtypes are known, subtype 1 (M1) has pro-inflammatory properties and is associated with tissue injury. Subtype 2 (M2) is associated with extracellular matrix remodelling and regulation of fibroblasts [34-36]. To evaluate the presence of macrophage subtype 1, the expression of interleukin 6 (ilo), nos 2 and $c d 86$ was determined. Nos 2 expression was a 2-fold lower in the Prolene group at 7 days follow-up, compared with the PDS group, but not significantly ( $p=0.932)$. Il6, $c d 86$ and nos 2 expression did not differ significantly between groups on both time points (see Fig. 5).

M2 polarisation is induced by interleukin 10 (illo) [36, 37] and macrophage subtype 2 is identified by an increased expression of i10, clec 10a, mrcl (cd206), cd163 and tgfbl. $T g f b l$ is typically expressed by the macrophage subtype $2 \mathrm{c}$ [36, 38-40]. A significantly higher expression of clec10a was found in the PDS group in comparison to Prolene at 7 days follow-up, namely 2.5 -fold higher $(p=0.007)$. At 21 days, no significant differences were detected and the clec10a expression in both the Prolene and Vicryl groups was comparable to the PDS group. Although not significantly, a twofold lower $i l 10$ expression was observed at 7 days follow-up in the Prolene group compared to the PDS group. $\mathrm{Mrcl}$ expression was 2.5-fold higher in the PDS group compared to Prolene and Vicryl at 7 days follow-up $(p=0.761)$ without reaching significance. The expression of tgfbl was a 1.4-fold lower in the Prolene group compared to the PDS group at 7 days follow-up $(p=0.619)$. A 3.3-fold and 1.4-fold lower expression of $c d 163$ was encountered in the Prolene and Vicryl groups, respectively, in comparison to the PDS group at 7 days follow-up $(p=0.117)$. The differences between groups in expression of $i l 10, m r c l, t g f b l$ and cdl63 were not significant at both time points (see Fig. 6).

\section{Vascularisation and fibroblast expression}

Furthermore, vascularisation was evaluated using vegfa, angtp 2 and nos 3 expression [41, 42], but no significant difference was detected between the groups regarding the expression (see Fig. 7). Collagen type 1 alpha 1 chain (collal), mmpl and fnl expression were a measure for fibroblast activity $[39,43]$. Prolene induced a 2.5 -fold lower expression of $m m p l$ at 7 days $(p=0.161)$ and twofold lower at 21 days $(p=0.180)$ in comparison to PDS. The comparison between groups regarding the expression of $f n 1$, colla 1 and $\mathrm{mmpl}$ did not reveal significant differences (see Fig. 7).

\section{Discussion}

The technique for closure of the abdominal wall is evolving to prevent incisional hernia development. Both the use of a small bite technique shows a reduction in the incidence of incisional hernia [22,23], as well as mesh placement after a laparotomy prevents incisional hernia in highrisk patients $[6,18]$. Reports in the literature regarding the optimal suture material for the closure of the abdominal wall are conflicting and focus on clinical outcomes, such as incisional hernia, suture sinuses and wound pain [4, $21,25,44]$.

The meta-analyses comparing non-absorbable sutures versus slow-absorbable sutures are confounded by factors in the study population; multiple types of incisions, emergency versus elective surgery and different suture materials or techniques are included. In the comparison between non-absorbable and slow-absorbable sutures, no differences are detected regarding the incisional hernia rate [21, 25]. However, slow-absorbable sutures do result in less wound pain and suture sinuses. This experiment intends to provide a pathophysiological foundation for the choice in suture material, hypothesising that improved healing of the abdominal wall leads to a reduction of the incidence of incisional hernia.

A fast-absorbable, slow-absorbable and non-absorbable suture material was compared in a rat model. In general, the foreign body reaction to biomaterials is subdivided into four phases: protein absorption, cell recruitment and adhesion, foreign body giant cell formation, and finally extracellular matrix formation and fibrotic encapsulation [45, 46]. In this experiment, the focus lies on the last three phases. Tissues were microscopically examined regarding the presence of different types of cells and collagen fibres. Consequently, the gene expression was determined using qPCR to explore vascularisation, fibroblast activity and macrophage polarisation. 
clec10a

II10

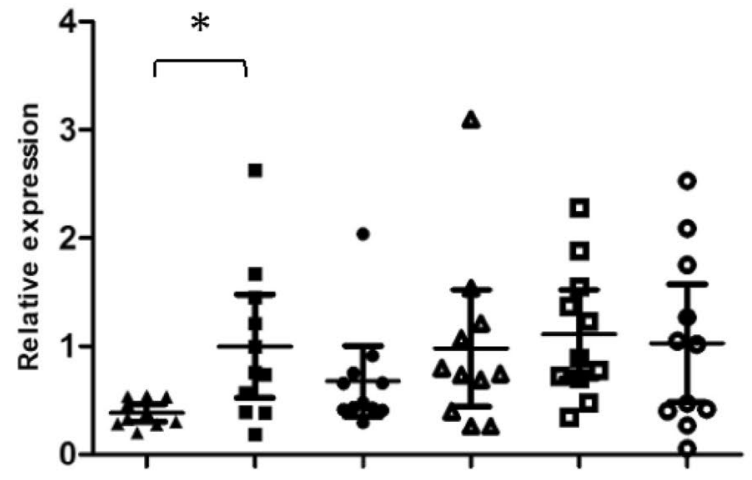

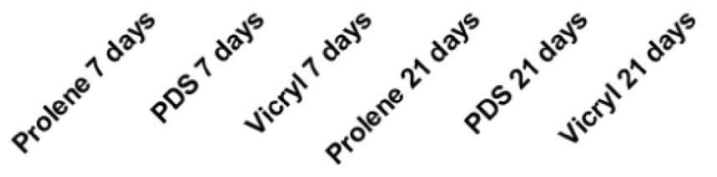

mrc1

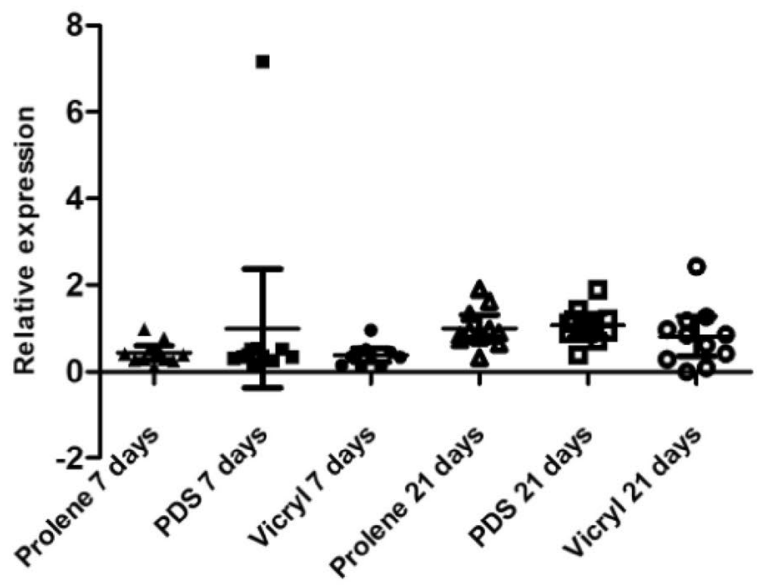

cd163
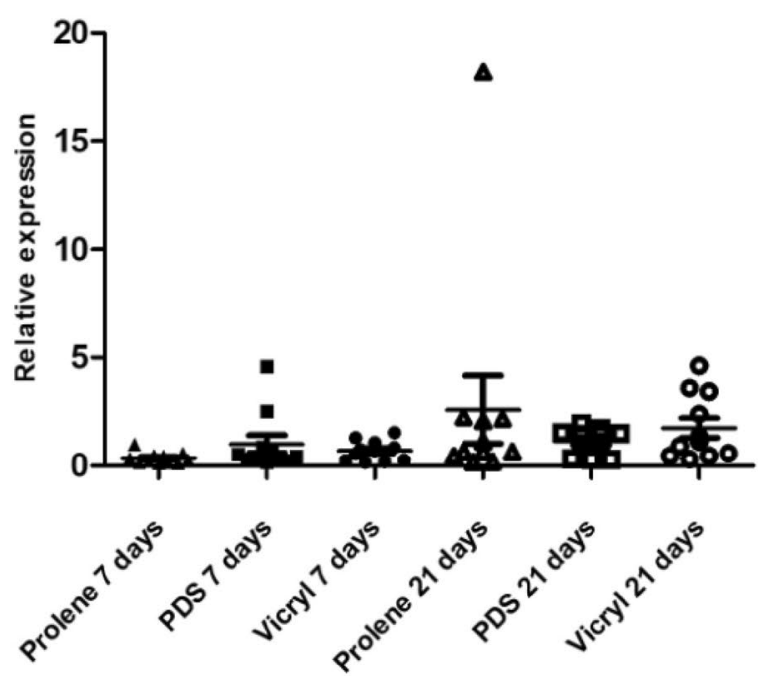

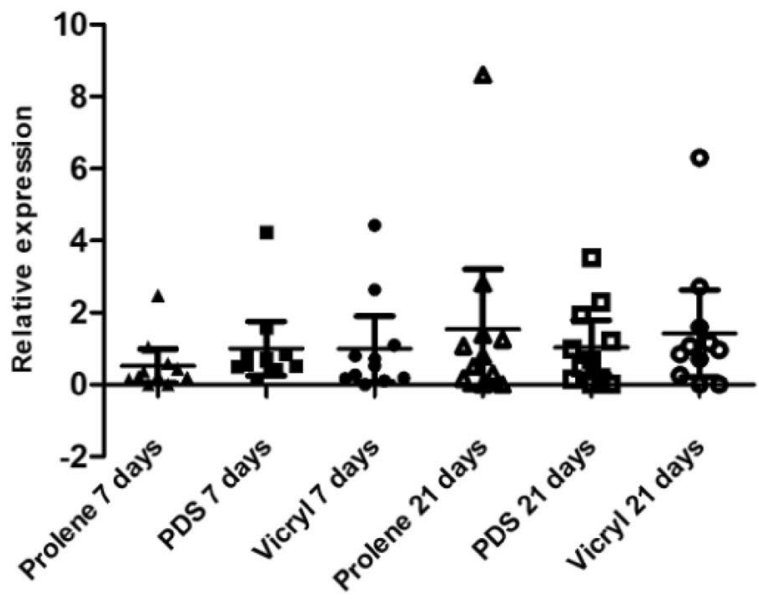

$\operatorname{tgfb1}$

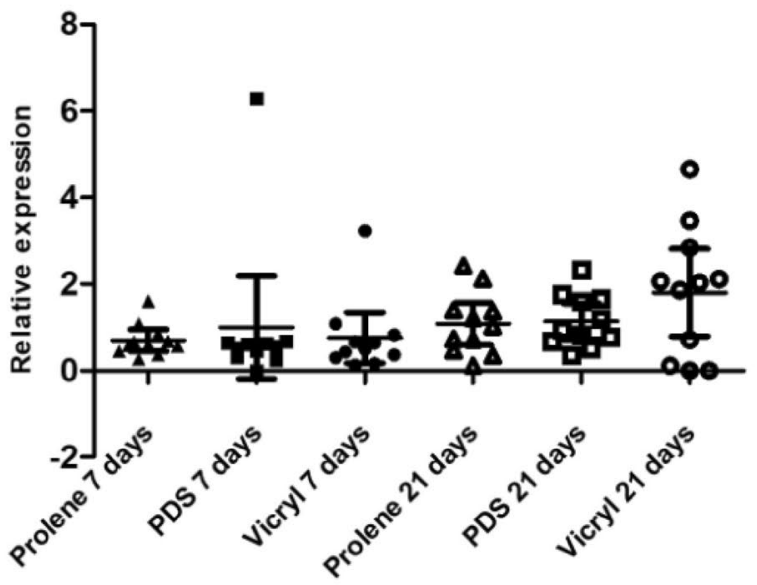


4Fig. 6 Scatter plots of relative expression of genes typically expressed by macrophage subtype 2 (clec10a, il10, mrcl, tgfbl, cd163) at 7 days and 21 days follow-up. The expression in the Vicryl and Prolene groups is depicted in relation to the PDS group, which was set as the norm. * $p=0.014$

The presence of macrophages was significantly higher in the Vicryl group after 21 days and simultaneously more foreign body giant cells were encountered compared to Prolene at 7 days and compared to PDS at 21 days. PDS and Vicryl are both absorbable sutures which rely on hydrolysis for degradation [47, 48]. Polylactic and polyglycolid acids, polymers both present in Vicryl, depend on phagocytosis by macrophages and especially foreign body giant cells for complete absorption [45, 49]. Vicryl elicits a stronger macrophage response in comparison to Prolene and PDS, which also results in more foreign body giant cells. The interaction between host and suture might play a role in its degradation and absorption. Although, in that case a similar finding would be expected in PDS. PDS differs from Vicryl in the polymer used and the fact that it is a monofilament suture in contrast to Vicryl which is braided [48]. These factors contribute to the difference in absorption rate between PDS and Vicryl, which, in the case of the faster absorption rate of Vicryl, can negatively affect the occurrence of incisional hernia. This concurs with clinically based results from a previous report, in which slow-absorbable sutures were considered superior regarding the incidence of incisional hernia compared to fast-absorbable sutures [20].

After determining the quantity of macrophages present, the polarisation between macrophage subtype 1 and 2 was evaluated using qPCR. Macrophage subtype 2 and its signalling play an essential role in liver regeneration, skeletal muscle healing and scar formation after injury of the skin [50-52]. Therefore, it was hypothesised that the macrophage subtype 2, which modulates extracellular matrix and activates fibroblasts [34-36], would have a positive effect on abdominal wall healing.

Clec10a expression, typical for macrophage subtype 2, was significantly higher in the PDS group compared to the Prolene group. This suggests a more dominant presence of macrophage subtype 2 in the healing process. A striking number of genes (illo, $m r c l, c d 163, t g f-b)$ typical for subtype 2 macrophages showed higher expression, although not significantly, when the abdominal wall was closed with PDS instead of Prolene after 7 days follow-up. The expression levels of the genes $m r c l$ and $c d 163$ were higher in the presence of PDS compared to Vicryl and Prolene after 7 days, without reaching significance. Nos2, typically expressed by the macrophage subtype 1 , and $m m p l$ showed a similar pattern. Although no significant differences could be detected, multiple genes typically expressed by subtype 2 macrophages were high in expression in the PDS group versus one subtype 1 specific gene. This could be interpreted as a distinct pattern, suggesting a dominant macrophage subtype 2 presence after abdominal wall closure with PDS. The effect disappears after 21 days of follow-up, which coincides with the progression of the healing process. The findings regarding macrophage polarisation concur with reports on scaffolds of different biomaterials, concluding a mainly antiinflammatory macrophage polarisation in reaction to PDS (polydioxanone). Regarding polypropylene and polylactic acid, both the pro-inflammatory and anti-inflammatory macrophage responses were reported [53].

The presence of different cell types in the analysed samples might result in various expression patterns, which could influence qPCR results. In addition, the macrophage polarisation is described as a spectrum rather than a black and white differentiation [54-56]. This may cause heterogeneity in the qPCR data and might limit the ability to reach a statistical difference. This might also be an argument for a larger sample size than calculated for this experiment to reach adequate power.

Recording the mere presence of macrophages no longer suffices in evaluation of the healing process of the abdominal wall and novel insights in macrophage polarisation need to be taken into account. Subtyping and activation of macrophages provide additional information on the regenerative process taking place in the abdominal wall. The anti-inflammatory subtype 2 macrophage is associated with tissue regeneration and is, therefore, assumed to have a positive effect on the healing process [34-36, 50-52]. The results of this experiment suggest a favourable macrophage response to PDS in comparison to Prolene and Vicryl, which in turn might benefit the regenerative capacity of the abdominal wall. This provides a new perspective on the dilemma of appropriate suture material in abdominal wall closure, which is of added value to the existing literature, which is mostly based on clinical outcomes.

\section{Conclusion}

This study provides an argument carefully supporting the use of PDS suture, in comparison to Vicryl or Prolene for closure of the abdominal wall. This is based on limited evidence indicating macrophage subtype 2 polarisation, a 
vegfa
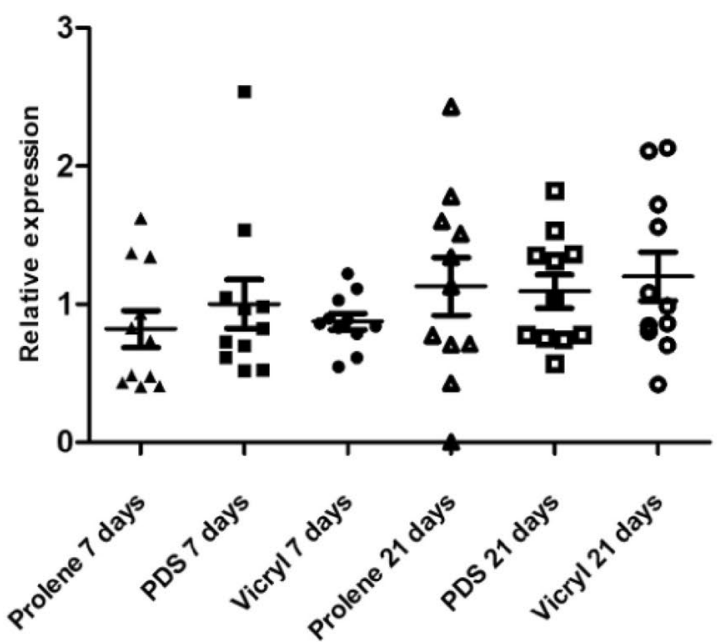

nos3
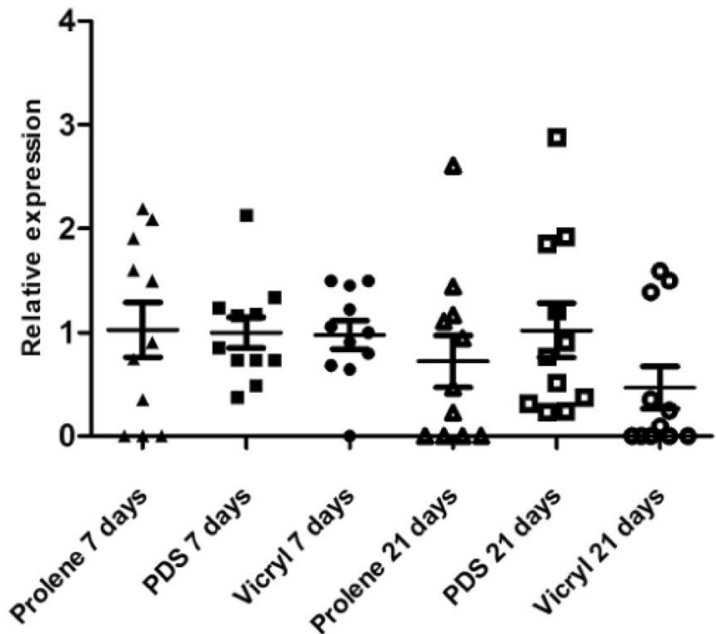

mmp1
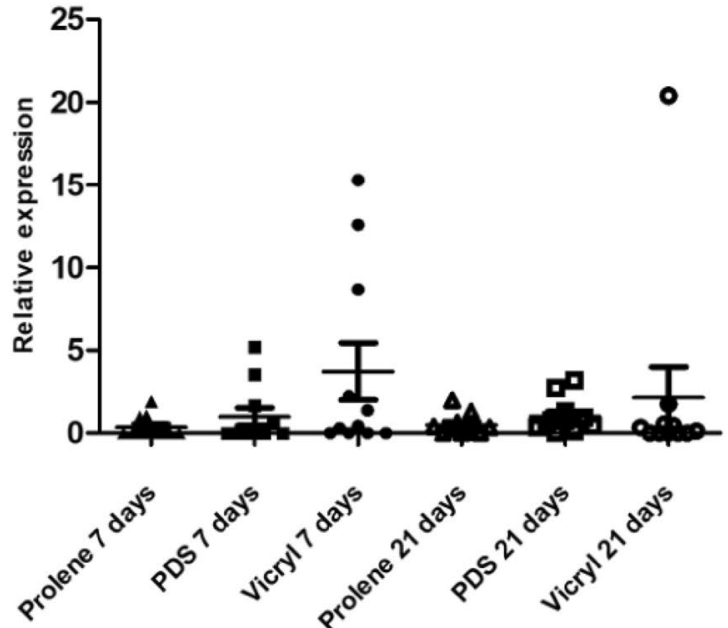

angtp2
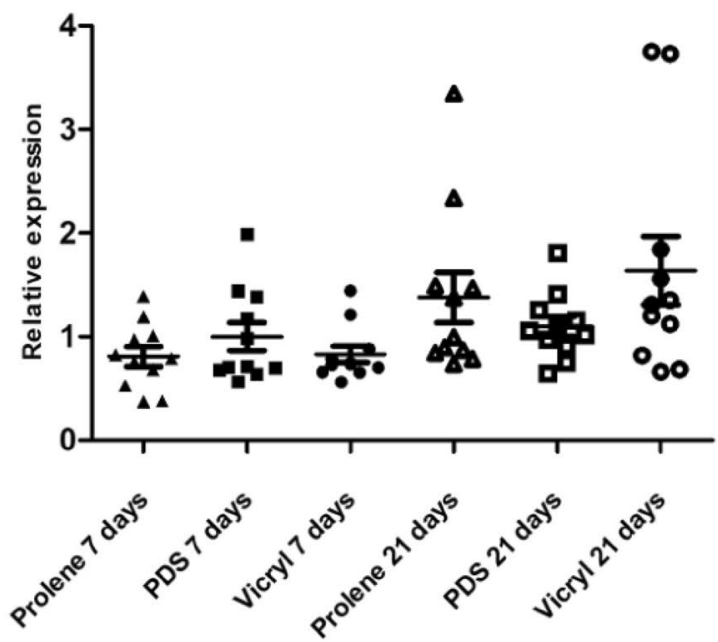

col1a1
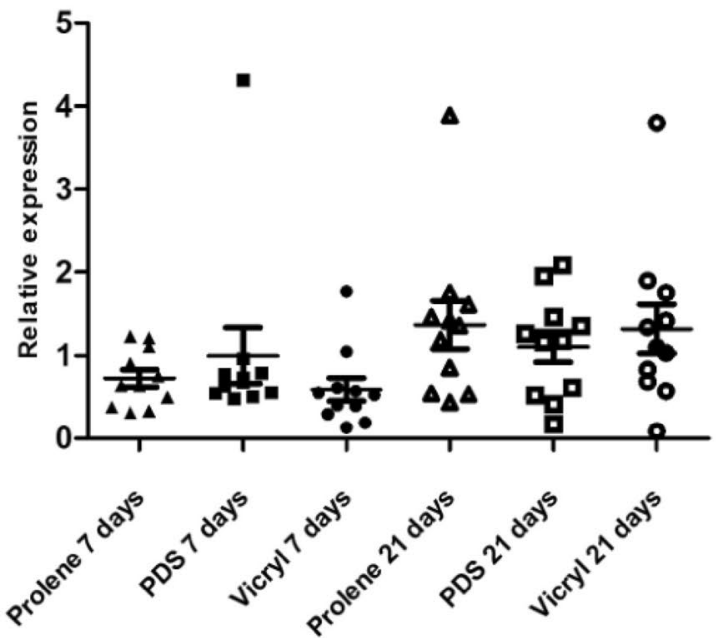

fn1

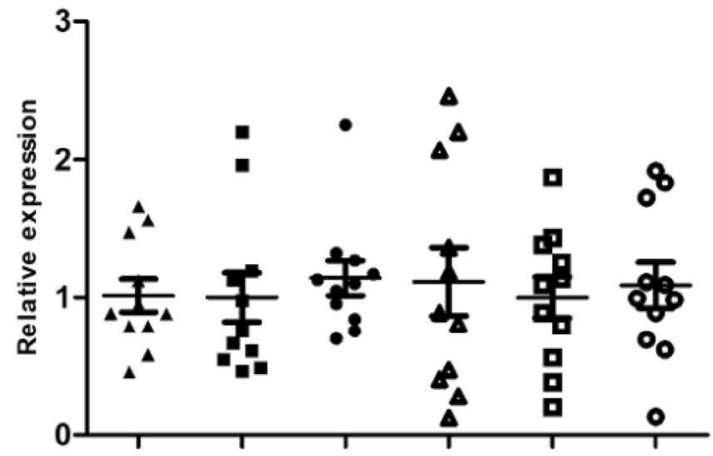

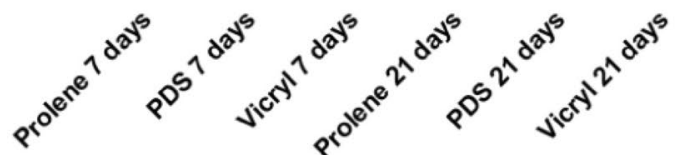


4Fig.7 Scatter plots of relative expression of genes typically expressed in case of vascularisation (vefga, angtp2 and nos3) and by fibroblast (collal, mmpl and fn1) at 7 days and 21 days follow-up. The expression in the Vicryl and Prolene groups is depicted in relation to the PDS group, which was set as the norm

favourable macrophage response which could play a role in the regeneration process of the abdominal wall.

Author contribution All authors contributed equally to this work with conception and design of the study, drafting and critical revision and final approval of the published article.

\section{Funding None.}

\section{Compliance with ethical standards}

Conflict of interest All authors have no conflicts of interest or financial ties to disclose.

Ethical approval The protocol was approved by the animal ethics committee of the Maastricht University conform the Dutch Experiments on Animals Act under protocol number 2014-089.

Human and animal rights All applicable international, national, and/or institutional guidelines for the care and use of animals were followed. This article does not contain any studies with human participants performed by any of the authors.

Informed consent Not required.

Open Access This article is distributed under the terms of the Creative Commons Attribution 4.0 International License (http://creativeco mmons.org/licenses/by/4.0/), which permits unrestricted use, distribution, and reproduction in any medium, provided you give appropriate credit to the original author(s) and the source, provide a link to the Creative Commons license, and indicate if changes were made.

\section{References}

1. Korenkov M, Paul A, Sauerland S et al (2001) Classification and surgical treatment of incisional hernia. Results of an experts' meeting. Langenbecks Arch Surg 386:65-73

2. Muysoms FE, Miserez M, Berrevoet F et al (2009) Classification of primary and incisional abdominal wall hernias. Hernia 13:407-414

3. van Ramshorst GH, Eker HH, Hop WC, Jeekel J, Lange JF (2012) Impact of incisional hernia on health-related quality of life and body image: a prospective cohort study. Am J Surg 204:144-150

4. Bosanquet DC, Ansell J, Abdelrahman T et al (2015) Systematic review and meta-regression of factors affecting midline incisional hernia rates: analysis of 14,618 patients. PLoS One 10:e0138745

5. Bloemen A, van Dooren P, Huizinga BF, Hoofwijk AG (2012) Comparison of ultrasonography and physical examination in the diagnosis of incisional hernia in a prospective study. Hernia 16:53-57

6. Jairam AP, Timmermans L, Eker HH et al (2017) Prevention of incisional hernia with prophylactic onlay and sublay mesh reinforcement versus primary suture only in midline laparotomies
(PRIMA): 2-year follow-up of a multicentre, double-blind, randomised controlled trial. Lancet 390:567-576

7. Antoniou GA, Georgiadis GS, Antoniou SA, Granderath FA, Giannoukas AD, Lazarides MK (2011) Abdominal aortic aneurysm and abdominal wall hernia as manifestations of a connective tissue disorder. J Vasc Surg 54:1175-1181

8. Henriksen NA, Helgstrand F, Vogt KC et al (2013) Risk factors for incisional hernia repair after aortic reconstructive surgery in a nationwide study. J Vasc Surg 57:1524-1530

9. Hoer J, Lawong G, Klinge U, Schumpelick V (2002) Factors influencing the development of incisional hernia. A retrospective study of 2983 laparotomy patients over a period of 10 years. Chirurg 73:474-480

10. Judge DP, Dietz HC (2005) Marfan's syndrome. Lancet 366:1965-1976

11. Kroese LF, Mommers EH, Robbens C, Bouvy ND, Lange JF, Berrevoet $\mathrm{F}$ (2018) Complications and recurrence rates of patients with Ehlers-Danlos syndrome undergoing ventral hernioplasty: a case series. Hernia 22:611-616

12. Uden A, Lindhagen T (1988) Inguinal hernia in patients with congenital dislocation of the hip. A sign of general connective tissue disorder. Acta Orthop Scand 59:667-668

13. Burger JW, Luijendijk RW, Hop WC, Halm JA, Verdaasdonk EG, Jeekel J (2004) Long-term follow-up of a randomized controlled trial of suture versus mesh repair of incisional hernia. Ann Surg 240:578-583 (discussion 83-5)

14. Flum DR, Horvath K, Koepsell T (2003) Have outcomes of incisional hernia repair improved with time? A population-based analysis. Ann Surg 237:129-135

15. Helgstrand F, Rosenberg J, Kehlet H, Strandfelt P, Bisgaard T (2012) Reoperation versus clinical recurrence rate after ventral hernia repair. Ann Surg 256:955-958

16. Luijendijk RW, Hop WC, van den Tol MP et al (2000) A comparison of suture repair with mesh repair for incisional hernia. N Engl J Med 343:392-398

17. Bevis PM, Windhaber RA, Lear PA, Poskitt KR, Earnshaw JJ, Mitchell DC (2010) Randomized clinical trial of mesh versus sutured wound closure after open abdominal aortic aneurysm surgery. Br J Surg 97:1497-1502

18. Muysoms FE, Detry O, Vierendeels T et al (2016) Prevention of incisional hernias by prophylactic mesh-augmented reinforcement of midline laparotomies for abdominal aortic aneurysm treatment: a randomized controlled trial. Ann Surg 263:638-645

19. Kohler A, Lavanchy JL, Lenoir U, Kurmann A, Candinas D, Beldi G (2018) Effectiveness of prophylactic intraperitoneal mesh implantation for prevention of incisional hernia in patients undergoing open abdominal surgery: a randomized clinical trial. JAMA Surg. https://doi.org/10.1001/jamasurg.2018.4221

20. Diener MK, Voss S, Jensen K, Buchler MW, Seiler CM (2010) Elective midline laparotomy closure: the INLINE systematic review and meta-analysis. Ann Surg 251:843-856

21. van't Riet M, Steyerberg EW, Nellensteyn J, Bonjer HJ, Jeekel J (2002) Meta-analysis of techniques for closure of midline abdominal incisions. Br J Surg 89:1350-1356

22. Deerenberg EB, Harlaar JJ, Steyerberg EW et al (2015) Small bites versus large bites for closure of abdominal midline incisions (STITCH): a double-blind, multicentre, randomised controlled trial. Lancet 386:1254-1260

23. Millbourn D, Cengiz Y, Israelsson LA (2009) Effect of stitch length on wound complications after closure of midline incisions: a randomized controlled trial. Arch Surg 144:1056-1059

24. Muysoms FE, Antoniou SA, Bury K et al (2015) European hernia society guidelines on the closure of abdominal wall incisions. Hernia 19:1-24

25. Sajid MS, Parampalli U, Baig MK, McFall MR (2011) A systematic review on the effectiveness of slowly-absorbable versus 
non-absorbable sutures for abdominal fascial closure following laparotomy. Int J Surg 9:615-625

26. Henriksen NA, Yadete DH, Sorensen LT, Agren MS, Jorgensen LN (2011) Connective tissue alteration in abdominal wall hernia. Br J Surg 98:210-219

27. Gilliver SC, Ashworth JJ, Ashcroft GS (2007) The hormonal regulation of cutaneous wound healing. Clin Dermatol 25:56-62

28. Mommers EH, Hong L, Jongen A, Bouvy ND (2018) Baseline performance of the ischaemic button model for induction of adhesions in laboratory rats. Lab Anim. https://doi. org/10.1177/0023677218773116

29. Schreinemacher MH, Emans PJ, Gijbels MJ, Greve JW, Beets GL, Bouvy ND (2009) Degradation of mesh coatings and intraperitoneal adhesion formation in an experimental model. Br J Surg 96:305-313

30. Schreinemacher MH, van Barneveld KW, Dikmans RE, Gijbels MJ, Greve JW, Bouvy ND (2013) Coated meshes for hernia repair provide comparable intraperitoneal adhesion prevention. Surg Endosc 27:4202-4209

31. van den Hil LCL, Vogels RRM, van Barneveld KWY et al (2018) Comparability of histological outcomes in rats and humans in a hernia model. J Surg Res 229:271-276

32. Vogels RR, van Barneveld KW, Bosmans JW et al (2015) Long-term evaluation of adhesion formation and foreign body response to three new meshes. Surg Endosc 29:2251-2259

33. Ye J, Coulouris G, Zaretskaya I, Cutcutache I, Rozen S, Madden TL (2012) Primer-BLAST: a tool to design target-specific primers for polymerase chain reaction. BMC Bioinform 13:134

34. Martin P, Leibovich SJ (2005) Inflammatory cells during wound repair: the good, the bad and the ugly. Trends Cell Biol 15:599-607

35. Wynn TA, Barron L (2010) Macrophages: master regulators of inflammation and fibrosis. Semin Liver Dis 30:245-257

36. Martinez FO, Gordon S (2014) The M1 and M2 paradigm of macrophage activation: time for reassessment. F1000Prime Rep 6:13

37. Murray PJ, Wynn TA (2011) Obstacles and opportunities for understanding macrophage polarization. J Leukoc Biol 89:557-563

38. Barros MH, Hauck F, Dreyer JH, Kempkes B, Niedobitek G (2013) Macrophage polarisation: an immunohistochemical approach for identifying M1 and M2 macrophages. PLoS One 8:e80908

39. Ploeger DT, Hosper NA, Schipper M, Koerts JA, de Rond S, Bank RA (2013) Cell plasticity in wound healing: paracrine factors of M1/M2 polarized macrophages influence the phenotypical state of dermal fibroblasts. Cell Commun Signal 11:29

40. Wolf MT, Dearth CL, Ranallo CA et al (2014) Macrophage polarization in response to ECM coated polypropylene mesh. Biomaterials 35:6838-6849

41. Fukumura D, Gohongi T, Kadambi A et al (2001) Predominant role of endothelial nitric oxide synthase in vascular endothelial growth factor-induced angiogenesis and vascular permeability. Proc Natl Acad Sci USA 98:2604-2609

42. Gale NW, Thurston G, Hackett SF et al (2002) Angiopoietin-2 is required for postnatal angiogenesis and lymphatic patterning, and only the latter role is rescued by Angiopoietin-1. Dev Cell $3: 411-423$

43. Klinge U, Zheng H, Si Z et al (1999) Expression of the extracellular matrix proteins collagen I, collagen III and fibronectin and matrix metalloproteinase- 1 and -13 in the skin of patients with inguinal hernia. Eur Surg Res 31:480-490

44. Hodgson NC, Malthaner RA, Ostbye T (2000) The search for an ideal method of abdominal fascial closure: a meta-analysis. Ann Surg 231:436-442

45. Anderson JM, Rodriguez A, Chang DT (2008) Foreign body reaction to biomaterials. Semin Immunol 20:86-100

46. Junge K, Binnebosel M, von Trotha KT et al (2012) Mesh biocompatibility: effects of cellular inflammation and tissue remodelling. Langenbecks Arch Surg 397:255-270

47. Bourne RB, Bitar H, Andreae PR, Martin LM, Finlay JB, Marquis F (1988) In-vivo comparison of four absorbable sutures: vicryl, Dexon Plus, Maxon and PDS. Can J Surg 31:43-45

48. Hochberg J, Meyer KM, Marion MD (2009) Suture choice and other methods of skin closure. Surg Clin N Am 89:627-641

49. Matlaga BF, Salthouse TN (1983) Ultrastructural observations of cells at the interface of a biodegradable polymer: polyglactin 910 . J Biomed Mater Res 17:185-197

50. Goh YP, Henderson NC, Heredia JE et al (2013) Eosinophils secrete IL-4 to facilitate liver regeneration. Proc Natl Acad Sci USA 110:9914-9919

51. Heredia JE, Mukundan L, Chen FM et al (2013) Type 2 innate signals stimulate fibro/adipogenic progenitors to facilitate muscle regeneration. Cell 153:376-388

52. Knipper JA, Willenborg S, Brinckmann J et al (2015) Interleukin-4 receptor alpha signaling in myeloid cells controls collagen fibril assembly in skin repair. Immunity 43:803-816

53. Boersema GS, Grotenhuis N, Bayon Y, Lange JF, BastiaansenJenniskens YM (2016) The effect of biomaterials used for tissue regeneration purposes on polarization of macrophages. Biores Open Access 5:6-14

54. Biswas SK, Mantovani A (2010) Macrophage plasticity and interaction with lymphocyte subsets: cancer as a paradigm. Nat Immunol 11:889-896

55. Edwards JP, Zhang X, Frauwirth KA, Mosser DM (2006) Biochemical and functional characterization of three activated macrophage populations. J Leukoc Biol 80:1298-1307

56. Stout RD, Jiang C, Matta B, Tietzel I, Watkins SK, Suttles J (2005) Macrophages sequentially change their functional phenotype in response to changes in microenvironmental influences. J Immunol 175:342-349

Publisher's Note Springer Nature remains neutral with regard to jurisdictional claims in published maps and institutional affiliations. 